\title{
Combining Multiple Host-Based Detectors Using Decision Tree
}

\author{
Sang-Jun Han and Sung-Bae Cho \\ Dept. of Computer Science, Yonsei University \\ 134 Shinchon-dong, Sudaemoon-ku, Seoul 120-749, Korea \\ \{sjhan, sbcho\}@cs.yonsei.ac.kr
}

\begin{abstract}
As the information technology grows interests in the intrusion detection system (IDS), which detects unauthorized usage, misuse by a local user and modification of important data, have been raised. In the field of anomaly-based IDS several artificial intelligence techniques are used to model normal behavior. However, there is no perfect detection method so that most of IDSs can detect the limited types of intrusion and suffers from its false alarms. Combining multiple detectors can be a good solution for this problem of conventional anomaly detectors. This paper proposes a detection method that combines multiple detectors using a machine learning technique called decision tree. We use conventional measures for intrusion detection and modeling methods appropriate to each measure. System calls, resource usage and file access events are used to measure user's behavior and hidden Markov model, statistical method and rule-base method are used to model these measures which are combined with decision tree. Experimental results with real data clearly demonstrate the effectiveness of the proposed method that has significantly low false-positive error rate against various types of intrusion.
\end{abstract}

Keyword: anomaly detection, decision tree, combining detectors

\section{Introduction}

Today's high reliance on computers raises concerns about the computer security. As the damage from security incidents increases, several tools and equipments become essential to computer systems. Intrusion detection is to find attacks exploiting illegal uses or misuses. An IDS is software to detect attacks exploiting illegal uses or misuses and modification of important data by analyzing system calls, system logs, activation time, and network packets of each operating system [1]. Generally, intrusion detection techniques can be divided into two groups according to the type of data they use: misuse detection and anomaly detection [2].

Misuse detection has the advantage that known attacks can be detected reliably with low false-positive error and economically. The shortcoming is that it cannot detect unknown attacks. Anomaly detection is better than misuse detection in terms of detecting novel attacks and its low false-negative error rate. 
However, it suffers from high false-positive error rate because unseen normal behaviors are considered as attacks. Another drawback of anomaly detection technique is that the detectable types of intrusion are limited according to the measures and modeling methods used.

In this paper, to overcome drawbacks of the conventional anomaly detection techniques, we propose an anomaly-based detection technique that combines multiple detectors. First of all, we develop four appropriate detection methods that use system call events, resource usage of process, file access events as the measure of normal behavior with appropriate modeling methods. Next, we combine these detectors using decision tree. The proposed detection method is expected better performance because it can model normal behaviors from various perspectives.

The rest of this paper is organized as follows. In Section 2, we give a brief overview of the related works. The overall design and detailed description of the proposed methods are presented in Section 3. Experimental results are shown in Section 4.

\section{Related Works}

Various techniques are used for anomaly-based intrusion detection. Expert system, statistics, artificial neural network and hidden Markov model (HMM) are widely used for modeling normal behaviors. The representative studies on anomaly detection are summarized in Table 1.

Statistics is the most widely used technique, which defines normal behavior by collecting data relating to the behavior of legitimate users over a period of time [3]. The representative IDS based on statistics is NIDES (Next-generation Intrusion Detection Expert Systems), which measures the similarity between a subject's long-term behavior and short term behavior for intrusion detection [8]. The detection rate is high because it can use various types of audit data

Table 1. The representative studies on intrusion detection

ES: Expert System, NN: Neural Network, ST: Statistics, HMM: Hidden Markov Model

\begin{tabular}{c|c|c|c|c|c|c}
\hline Organization & Name & Period & ES & NN & ST & HMM \\
\hline AT\&T & ComputerWatch [4] & $1987-1990$ & X & & & \\
\hline UCDavis & NSM [5] & $1989-1995$ & & & X & \\
& GrIDS [6] & $1995-$ & X & & & \\
\hline SRI International & IDES [7] & $1983-1992$ & & & X & \\
& NIDES [8] & $1992-1995$ & & & X & \\
& EMERALD [9] & $1996-$ & & & X & \\
\hline CS Telecom & Hyperview [10] & $1990-1995$ & X & X & & \\
\hline Univ. of New Mexico & C. Wrannder et. al [11] & 1995 & & & X & X \\
\hline Yonsei Univ. & Park and Cho [12] & $1999-$ & & & & X \\
\hline
\end{tabular}


and detect intrusion based on the previous experimental data. The shortcoming is that it is not sensitive to some behavior and detectable types of intrusion are limited.

Hyperview of CS Telecom uses neural network for intrusion detection [10]. It consists of 2 modules: neural network and expert system. The neural network in Hyperview uses temporal sequence of audit data as inputs and 60 types of audit data. It has the advantage of easy representation of nonlinear relationship between input and output. The defects of neural networks are that its computational load is very heavy and it is difficult to interpret the relationship between inputs and outputs.

An HMM is useful technique because it is good for modeling system call sequences. The representative study is the technique proposed by $\mathrm{C}$. Warender of New Mexico University [11]. It uses system call audit trails to measure normal behaviors. While HMM produces better performance in modeling system call events than other methods, it requires very long time for modeling normal behaviors. The solution for this problem might be to improve the performance of computer system or to reduce the training data. The technique that reduces audit trails by filtering audit trails from abstracted information around the change of privilege flows can save the computational costs significantly while maintaining good performance [12].

\section{Proposed Method}

Among the intrusion types that frequently occur, buffer overflow, S/W security error, configuration error and denial of service attacks are prevalent on host. According to the hacking trends in May, June, and July 2002 reported by CERTCC the majority of attacks occurred is buffer overflow. Recently massive access to internet raises the issue of denial of service attack. This paper focuses on the two intrusion types to develop sophisticated detection method.

In this paper, we use common measures for host-based detection system: system call event, file system information and resource usage of process. Though there are several methods for modeling each measure, we select modeling methods appropriate to each measure considering the relationship between the characteristics of intrusion traces and the power of modeling methods. However, because each intrusion type leaves anomalies at individual measure, there are undetectable intrusion types in each measure and modeling method. To overcome this drawback, it is necessary to remedy shortcomings of each method through combining multiple detectors. We construct decision tree that combines multiple measure models and detect intrusions with it. The general architecture of the proposed method can be seen in Fig 1.

\subsection{HMM with System Call Events}

Sun Microsystem's Basic Security Module (BSM) which is auditing facility for Solaris provides an adequate representation of the behavior of the program be- 


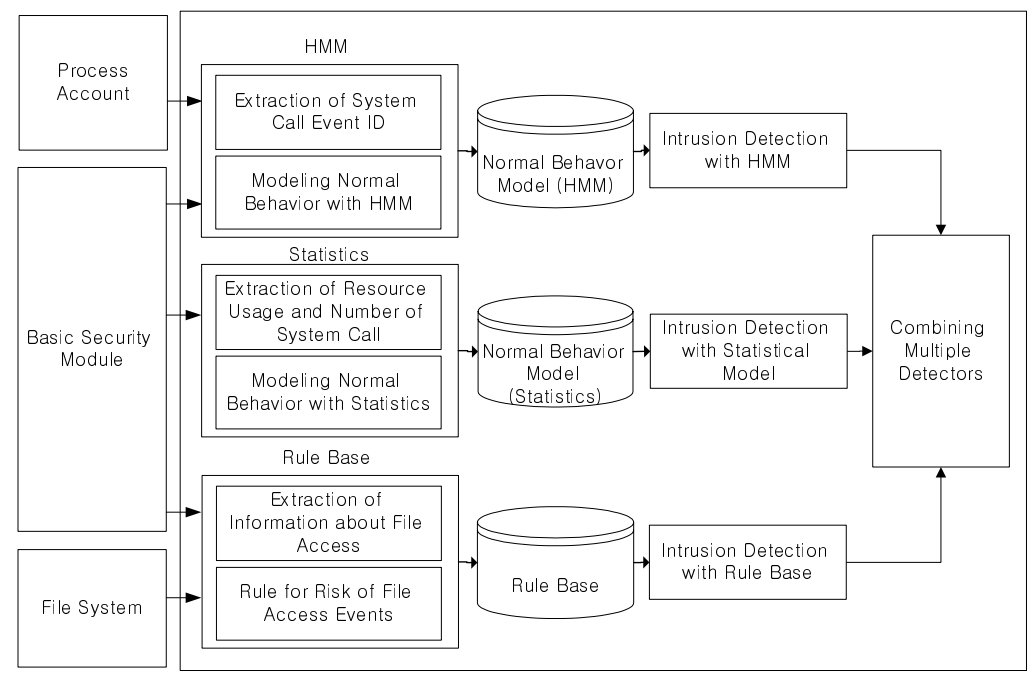

Fig. 1. Overall structure of the proposed method

cause any privileged activities that might be generated by a program are captured by BSM. Usually audit trail from BSM consists of several measures. The victim process of a certain attack generates system call events that are significantly different from events generated at normal situation. Thus, we can detect intrusions effectively by building the model of system call events from normal situation and noting significant deviation from the model. In this paper, for modeling system call event sequences, we use HMM that is widely used for speech recognition because it is very useful for modeling sequence information. HMM can be successfully applied to modeling system call event sequences [13].

Intrusion detection with HMM consists of two phases: normal behavior modeling and anomaly detection. The first phase is normal behavior modeling, which is determining HMM parameters to maximize the probability $\operatorname{Pr}(O \mid \lambda)$ with which input sequence is generated out of given normal behavior model. Because no analytic solution is known for it, an iterative method called Baum-Welch reestimation is used. Anomaly detection, the second phase, matches current behavior against the normal behavior model, and calculates the probability with which it is generated out of the model. Forward-backward procedure is used for this purpose [14]. The probability is used to decide whether normal or not with a threshold.

\subsection{Statistics with Resource Usage and System Call Events}

Most of Unix-based operating systems serve Process Account (PACCT) as audit data. It provides the resource usage of processes: CPU time, memory usage, I/O usage, etc. Denial of service attack sharply raises the resource usage of victim 
process. This type of attack can be detected by noting processes that show unusual resource usage compared with normal behavior using PACCT audit data.

PACCT audit data is useful to detect DoS attacks. Unfortunately, we cannot detect attack type that targets resource not recorded to PACCT. For example, attack that consumes process table leaves no anomalies in PACCT. However, it unusually generates large amount of system call events. In this case, we can detect that by noting process that generates unusual number of system call events.

In this paper, we use statistical technique to model normal resource usage and the number of system call events. This statistical approach is a modified method that has used in NIDES. For each audit record generated by user, we generate a single test statistic value denoted by $T$ that summarizes the degree of abnormality in the user's behavior in the near past. Large values of $T$ indicate abnormal behavior, and values close to zero indicate normal behavior. In the case of PACCT, the $T$ statistic is a summary judgment of the abnormality of measures in PACCT. We denote the individual abnormality of measures by $S$, each of which measures the degree of abnormality of behavior with respect to specific features such as CPU time, memory usage and I/O usage. $T$ statistic has been set equal to the weighted sum of the $S$ statistics as follows:

$$
T=a_{1} s_{1}+a_{2} s_{2}+\ldots+a_{n} s_{n}
$$

where $s_{n}$ is the $S$ score of each measure and $a_{n}$ is the weight to each measure.

Each $S$ statistics is derived from a corresponding statistic called $Q$. In fact, each $S$ statistic is a normalizing transformation of the $Q$ statistic so that the degree of abnormality for different types of features can be added on a comparable basis. The value of $Q$ corresponding to the current audit record represents the number of audit records that are arrived in the recent past. In order to transform $Q$ to $S$, we have built a normal historical profile of all previous values of $Q$ and compare the current value of $Q$ with this normal profile to determine if the current value is anomalous.

Small value of $Q$ indicates a recent past that is similar to historical behavior, and large values of $Q$ indicates a recent past that is not similar to historical behavior. Given that $k$ is an index of appropriate audit records, $t_{k}$ is the time that elapses between the $k$ th and the most recent audit records, $r$ is the decay rate and $D_{k}$ is the change that occurs between the $(k+1)$ st and $k$ th appropriate audit records, $Q$ is defined as follows [15]:

$$
Q \sum_{k \geq 1} D_{k} \times 2^{-r t_{k}}
$$

\subsection{Rule-Base with File Access Events}

Generally, attacks tend to access files of which the security level is high. For example, it executes file which has a SETUID privilege to acquire root privilege 
or attempts to read files owned by root to destroy the computer system or obtain a critical data. Owing to this tendency of abnormal behavior, it is adequately suspicious behavior for ordinary user to access files which have higher security level.

The best-known security policy related with file access is the Bell-LaPadula (BLP) model which has been formulated by Bell and LaPadula [16]. In an access to some information, there are three primary elements: subject, object and access attribute. The subject corresponds to user or program, the object corresponds to files and the access attribute corresponds to the kind of access model: read, write, execute, etc. In order to determine if a specific access mode is allowed, the BLP model compares the clearance of a subject with the classification of the object and determination is made as to whether the subject is authorized for the specific access mode [17]. The BLP model includes several rules referenced frequently. One of them is no read up rule, which allows access if the current level of the subject dominates the level of the object. It prevents user from accessing information for which they are not allowed to access.

In this paper, we audit file access and evaluate the risk of access event by analyzing the content of that. The security levels of subject and object are divided into 4 categories: root, administrator, ordinary user and guests. The risk of access event is evaluated to one of 21 levels according to the difference of security levels between subject and object. The access event of which the difference between two levels is bigger has higher risk. If the level of object is lower than that of subject the risk is not increased due to the security level difference. If the access event contains file that allows more operations, it has higher risk. For example, when the file mode is 755 the access event is riskier than that of 744 .

\subsection{Combining Multiple Detectors}

In this paper, we have proposed an intrusion detection technique that combines multiple detectors in order to increase detectable attack types and reduce falsepositive error rate. We use decision tree to combine multiple detectors. Decision tree is one of the most widely used methods for inductive inference. Constructing decision tree is to build up tree data structure that predicts the class of a case in terms of the attribute values of the case. Each node of tree specifies a test of some attribute of the case and each branch corresponds to one of the possible values for this attribute. The tree-construction algorithm infers trees by growing them from the root downward and selecting the next best attribute for each new branch added to the tree.

We have used Quinlan's C4.5 which is a well-known decision tree generation algorithm [18]. C4.5 uses an information-theoretical approach based on the energy entropy. It builds the decision tree using a divide-and-conquer approach: select an attribute, divide the training set into subsets characterized by the possible values of the attribute, and follow the same partitioning procedure recursively with each subset until no subset contains objects from more than one 
class [19]. The single class subsets correspond them to the leaves. The entropybased criterion that has been used for the selection of the attribute is called the gain ratio criterion.

Let $T$ be the set of classes of cases associated at the node. At first, the frequency of cases in $T$ which belongs to some class $C_{j}$ is computed. If there is a class $C_{j}$ whose frequency is 1 (i.e. all cases belongs to cases) or higher than some value a leaf node is made with associated class $C_{j}$. If there is no class that has high frequency the information gain of each attribute is calculated. The information gain is a measure of the effectiveness of an attribute in classifying the data [20]. The attribute which has maximum information gain is selected. If the selected attribute is continuous, the tree branches into binary nodes with a certain threshold. If the selected attribute is discrete, the tree branches for each possible value of that attribute. Let $T^{\prime}$ one of the sets that are produced by the test on the selected attribute and the same operation is applied recursively on each non-empty $T^{\prime}$. If $T^{\prime}$ is empty, the child node is a leaf. The overall process of tree-construction algorithm is given as follows:

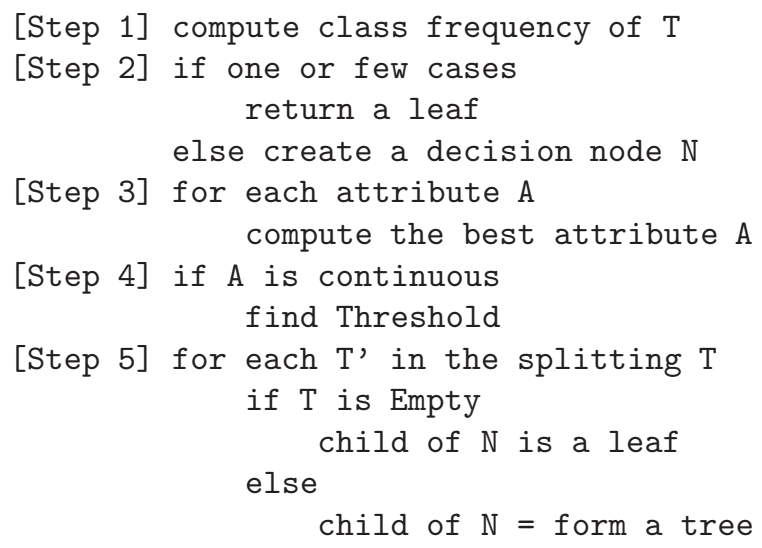

An audit data set that contains labeled attacks mixed with normal behavior is collected for training decision tree. Each of the four detectors evaluates this data set and the evaluation result constitutes the decision tree training data that conforms to data format of the decision tree generator. Each record of training data consists of four continuous real value (evaluation results) and labeled as two classes: attack and normal. The decision tree generator constructs decision

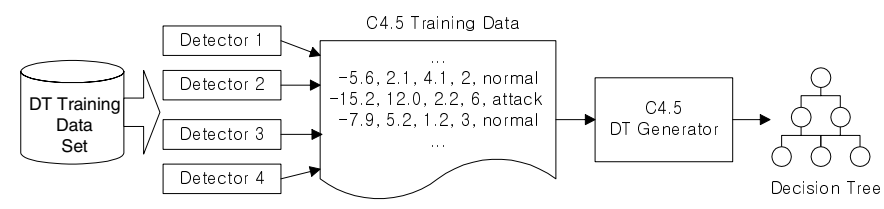

Fig. 2. Constructing the decision tree 
tree and generated tree is used to combine multiple detectors. Overall process of constucuting decision tree is shown in Fig. 2.

The decision tree is constructed with decision tree training data set as shown in Fig. 3. 8 rules are generated by decision tree generator since each leaf node corresponds to one rule. The rules that are more operative (frequently used to classify items) are given as follows:

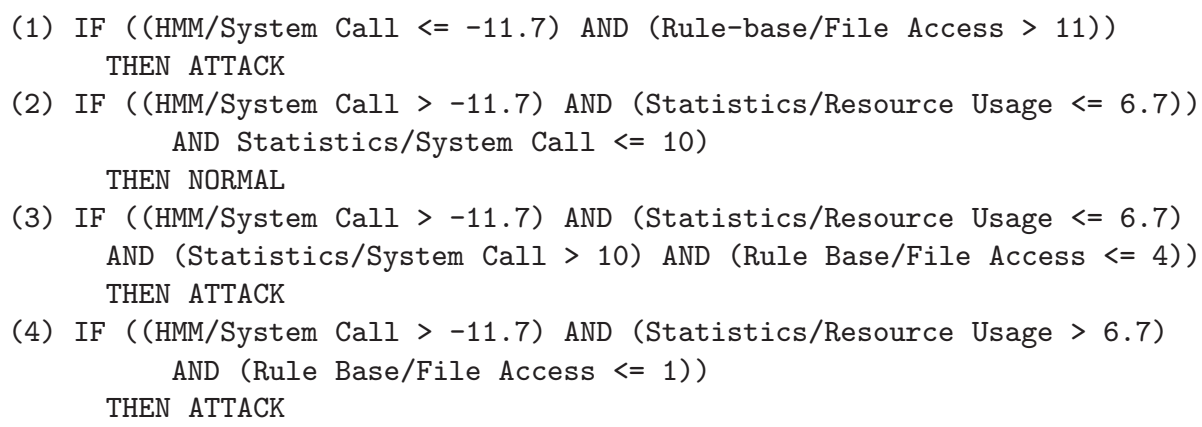

Rule 1 classifies the process that produces suspicious system call sequences and accesses risky files as attack. This rule detects most of buffer overflow attacks because it generates abnormal system call event sequences and accesses files that have SETUID previledge to obtain root privilege. Rule 2 discriminates most of normal behaviors because it classifies the processes that generate normal system call sequences and show ordinary resource usage and system call as normal behavior. Rule 3 and 4 detect denial of service type attacks. Rule 3 classifies as attack processes that show ordinary resource usage and system call sequences but use large amount of system call such as process table consuming attack. Rule 4 determines processes that shows unusual resource usage such as disk and memeory consumption as attack. Both of rules that detect the denial of services attacks include the 'smaller than' condition with the risk of file access because this type of attack does not require to access the risky files.

\section{Experiments}

We have collected normal behaviors from six graduate students for 2 weeks using Solaris 7 operating system. They have mainly used text editor, compiler and programs of their own writing. Total 13 megabytes (160,448 records) of BSM audit data and 840 kilobytes of PACCT audit data have been collected from 16,470 commands. We also collect audit data that contain labeled attacks for testing in the same operating system. It contains 9 cases of u2r buffer overflow intrusion and 4 cases of denial of services. Audit data for constructing decision tree have also been collected. It contains 28 cases of labeled attack mixed with normal behavior. The attacks used in our experiments are as shown in Table 2.

In this paper, the experiments have been conducted in three phases. At first, we have built behavior profile of normal data and tested the performance of each detector. Next, we construct decision tree with decision tree training algorithm to 


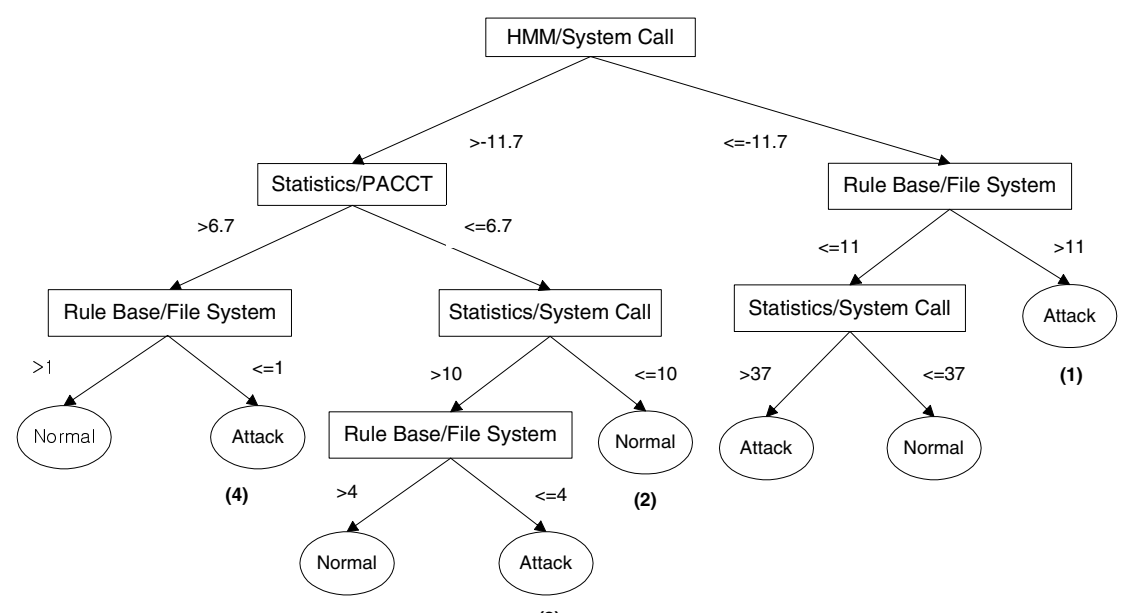

(3)

Fig. 3. The constructed decision tree

Table 2. The attacks used in our experiments

\begin{tabular}{c|c}
\hline Attack Type & Attack Name \\
\hline Buffer Overflow & $\begin{array}{c}\text { kcms_configure buffer overflow vulnerability } \\
\text { lpset -r buffer overflow vulnerability } \\
\text { xlock heap buffer overflow vulnerability }\end{array}$ \\
\hline Denial of Service & $\begin{array}{c}\text { Consumption of Disk } \\
\text { Consumption of Process Table } \\
\text { Consumption of Memory }\end{array}$ \\
\hline
\end{tabular}

combine multiple detectors. The performance of combined method is evaluated with test data set. The overview of the experiment is shown in Fig. 4.

\subsection{Performance Metrics}

The important metrics in evaluating the performance of IDS are detection rate and false-positive error rate. We calculate the detection rate as the rate of detected intrusions. Each attack counts as one detection if at least one process which is used in attack process is evaluated as intrusion. The false-positive error rate is calculated as the rate of mis-classified processes.

In the experiments, we visualize the performance of detection method through ROC (Receiver Operating Characteristics) curve. An ROC curve depicts the changes in attack detection rate and false-positive error rate as modifiable parameter is changed. In this paper, evaluation threshold is used as a modifiable parameter. A desirable intrusion detection system must show a high detection 


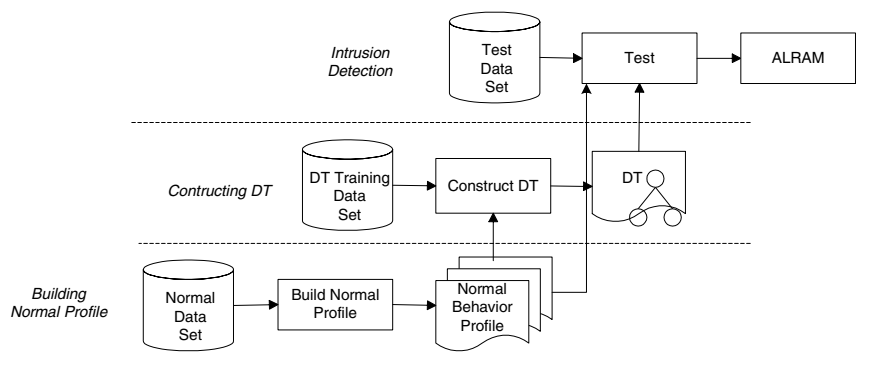

Fig. 4. Overview of the experiment

rate at low false-positive error rate. In ROC curve, top-left curve is more desirable.

We use discriminability to measure numerically the performance of a detection method and efficiency to compare integration methods with others. The discriminability is a measure of the average intensity difference perceived by an observer between samples including the signal and samples not including a signal. It has higher value at high detection rate and low false-positive error rate [21]. Generally, it has been noted as $d^{\prime}$ and defined as follows:

$$
d^{\prime}=z(H)-z(F)
$$

where $\mathrm{z}$ is the inverse of the normal distribution function, $H$ is detection rate and $F$ is false-positive error rate.

\subsection{Experimental Results}

At first, we have conducted experiments without the integration of detection methods in order to identify the characteristics of each method and find optimal parameters of each method. The experimental result of individual detection methods does not show good performance owing to the characteristics of measure and modeling methods. We have compared the detection methods with the best parameters: The number of states is 3 and the length of input sequence is 8 in HMM and the weight ratio to resource usage is 2:1:2 (CPU time: memory usage: I/O usage). The false-positive error rate has been sharply raised after a certain degree of detection rate as shown in Fig. 5 .

The experiment with the proposed method using the constructed decision tree is conducted using the test data set with the parameters of each method that show the best performance in the preliminary experiments. We have compared false-positive error rate and discriminability of each method at $100 \%$ detection rate as shown in Table 3 . The result shows the combined detection method dramatically reduces the false-positive error rate. 


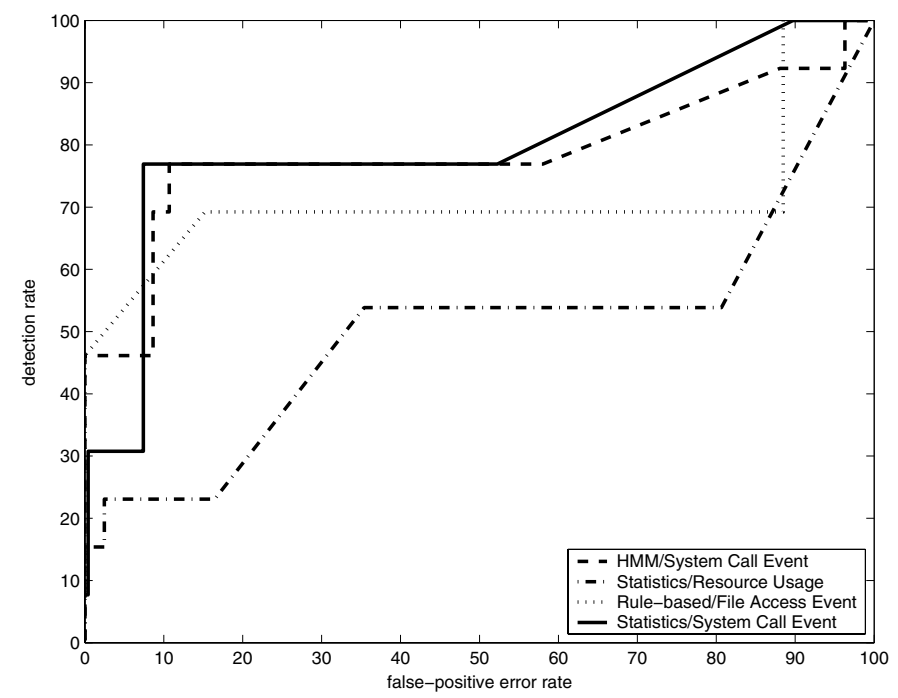

Fig. 5. ROC for individual detection methods with best parameters

Table 3. A comparison of each detection method

\begin{tabular}{c|c|c|c}
\hline Method & Detection rate & F-P error rate & d' \\
\hline HMM/System Call Event & $100 \%$ & $99.177 \%$ & 1.866 \\
Statistics/Resource Usage & $100 \%$ & $80.658 \%$ & 3.400 \\
Statistics/System Call Event & $100 \%$ & $99.177 \%$ & 1.866 \\
Rule base/File Access Event & $100 \%$ & $88.477 \%$ & 3.066 \\
Combining with Decision Tree & $100 \%$ & $0.412 \%$ & 5.733 \\
\hline
\end{tabular}

\section{Conclusions and Future Work}

In this paper, we have presented effective modeling methods for three audit data and proposed a novel intrusion detection technique that integrates the detection methods. The proposed method uses multiple measure and modeling methods and combines the results of individual detectors using decision tree to overcome the drawbacks of the conventional anomaly detection techniques.

We evaluate the performance of each detection method and compare the results with proposed method. The proposed method shows $0.412 \%$ false-positive error rate at $100 \%$ detection rate whereas the other methods show more than $80 \%$ false-positive error rate at the same detection rate. It indicates that we can overcome the drawbacks by integrating several methods.

Decision tree has the advantage that the user can intuitively and visually understand rules which are generated by decision tree that combines multiple detectors autonomously. In the future, it is needed to perform experiments with larger data set such as the data set of DARPA Intrusion Detection System 
Evaluation program for more accurate evaluation of the proposed method. In addition, it is also needed to consider the computaional cost for using multiple models.

\section{Acknowledgements}

This research was supported by University IT Research Center Project.

\section{References}

1. H. S. Vaccaro and G. E. Liepins, "Detection of anomalous computer session activity," In Proceedings of IEEE Symposium on Research in Security and Privacy, pp. 280-289, 1989. 208

2. S. Axelsson, "Intrusion detection systems: A survey and taxonomy," Technical Report 99-15, Department of Computer Engineering, Chalmers University, March 2000. 208

3. E. Biermann, E. Cloete and L.M. Venter, "A comparison of intrusion detection systems," Computers \& Security, vol 20, no. 8, pp. 676-683, December 2001. 209

4. C. Dowel and P. Ramstedt, "The computer watch data reduction tool," In Proceedings of the 13th National Computer Security Conference, pp. 99-108, Washington DC, USA, October 1990. 209

5. T. Heberlein, G. Dias, K. Levitt, B. Mukherjee, J. Wood, and D. Wolber, "A network security monitor," In Proceedings of the 1990 IEEE Symposium on Research in Security and Privacy, pp. 296-304, Los Alamitos, CA, USA, 1990. 209

6. S. Staniford-Chen, S. Cheung, R. Crawford, M. Dilger, J. Frank, J. Hoagland, K Levitt, C. Wee, R. Yip, and D. Zerkle, "GrIDS-A graph based intrusion detection system for large networks," In Proceedings of the 19th National Information Systems Security Conference, vol. 1, pp. 361-370, October, 1996. 209

7. T.F. Lunt, A. Tamaru, F. Gilham, R. Jagannathan, C. Jalali, and P. G. Neuman, "A real-time intrusion-detection expert system (IDES)," Technical Report Project 6784, CSL, SRI International, Computer Science Laboratory, SRI International, February 1992. 209

8. D. Anderson, T. F. Lunt, H. Javits, A. Tamaru and A. Valdes, "Detecting unusual program behavior using the statistical components of NIDES," NIDES Technical Report, SRI International, May 1995. 209

9. P. A. Porras and P. G. Neumann, "EMERALD: Event monitoring enabling responses to anomalous live disturbances," In Proceedings of the 20th National Information Systems Security Conference, pp. 353-365, Baltimore, Maryland, USA, October 1997. 209

10. H. Debar, M. Becker and D. Siboni, "A neural network component for an intrusion detection system," In Proceedings of 1992 IEEE Computer Society Symposium on Research in Security and Privacy1, pp. 240-250, Oakland, CA, May 1992. 209, 210

11. C. Warrender, S. Forrest and B. Pearlmutter, "Detecting intrusion using calls: Alternative data models," In Proceedings of IEEE Symposium on Security and Privacy, pp. 133-145, May 1999. 209, 210

12. S.-B. Cho and H.-J. Park, "Efficient anomaly detection by modeling privilege flows with hidden Markov model," Computers ES Security, vol. 22, no. 1, pp. 45-55, 2003. 209, 210 
13. S.-B. Cho, "Incorporating soft computing techniques into a probabilistic intrusion detection system," IEEE Transactions on Systems, Man and Cybernetics-Part C:Applications and Reviews, vol. 32, no. 2, pp. 154-160, May 2002. 211

14. L. R. Rabiner and B. H. Juang, "An introduction to hidden Markov models," IEEE ASSP Magazine, pp. 4-16, January 1986. 211

15. H.S. Javitz and A. Valdes, "The SRI IDES statistical anomaly detector," NIDES Technical Report, SRI International, 1991. 212

16. Bell, D. E. and LaPadula, L. J., "Secure computer systems: Unified exposition and multics interpretation," Mitre Technical Report ESD-TR-75-306, Mitre Corporation, March 1976. 213

17. A. G. Amoroso, Fundamentals of Computer Security Technology, PTR Prentice Hall, New Jersy, 1994. 213

18. J. R. Quinlan, C4.5: Programs for Machin Learning, Morgan Kaufmann, 1993. 213

19. S. Ruggieri, "Efficient C4.5," IEEE Transactions on Knowledge and Data Engineering, vol. 14, no. 2, March 2002. 214

20. T. Mitchell, Machine Learning, McGraw-Hill, 1997. 214

21. N. A. Macmillan and C. D. Creelman, Detection Theory: A User's Guide, Cambridge University Press, Cambridge, 1991. 217 